\title{
Localization of targets across saccades: Role of landmark objects
}

\author{
Heiner Deubel \\ Department of Psychology, Ludwig-Maximilians-Universität, Germany
}

\begin{abstract}
Saccadic eye movements are required to bring different parts of the visual world into the foveal region of the retina. With each saccade, the images of the objects drastically change their retinal positions - nevertheless, the visual world appears continuous and does not seem to jump. How does the visual system achieve this continuous and stable percept of the visual world, despite the gross changes of its retinal projection that occur with each saccade? The present paper argues that an important factor of this type of space constancy is formed by the reafferent information, i.e., the visual display that is found when the eyes land. Three experiments demonstrate that objects present across the saccade can serve as landmarks for postsaccadic relocalization. The basic experimental manipulation consisted of a systematic displacement of these landmark objects during the saccade. The effectiveness of the landmarks was determined by analysing to what degree they modify the perceived shift of a small saccade target that was blanked for $200 \mathrm{~ms}$ during and after the saccade. A first experiment studied the spatial range where objects become effective as landmarks. The data show that landmarks close to the saccade target and horizontally aligned with the target are specifically effective. The second experiment demonstrates that postsaccadic localization is normally based on relational information about relative stimulus positions transferred across the saccade. A third experiment studied the effect of a prominent background frame on transsaccadic localization; the results suggest that background structures contribute only little to transsaccadic localization.
\end{abstract}

When the eye fixates an area in the visual field, the visual system has only access to a very restricted part of the scene, since high resolution and elaborated processing capabilities are limited to a narrow region around the central fovea. Therefore, saccadic eye movements are required that bring different regions of the world into the foveal region of the retina. However, although saccades provide the visual system with new information, they also induce several

Please address correspondence to: Heiner Deubel, Department Psychologie, LudwigMaximilians-Universität, Leopoldstrasse 13, D-80802 München, Germany.

Email: Deubel@psy.uni-muenchen.de

This study was supported by the Deutsche Forschungsgemeinschaft (DE 336/2).

(C) 2004 Psychology Press Ltd

http://www.tandf.co.uk/journals/pp/13506285.html

DOI:10.1080/13506280344000284 
problems that the perceptual system must solve. A first problem results from the high retinal velocity during a saccade, which leads to a smearing of the retinal projection. Nevertheless, the visual world appears continuous and we do not perceive saccade-induced "wipe-outs" of the visual information. A second problem arises from the fact that the images of the objects in the world drastically change their retinal positions during each saccade. However, the visual world does not seem to jump and we do not become disoriented with each saccade. This has lead to the question of how the visual system achieves this continuous and stable percept of the visual world, despite the gross changes of its retinal projection that occur with each saccade.

Von Helmholtz (1866/1962) provided one of the first accounts of the problem of space constancy. He assumed that constancy of visual direction is maintained by combining the image motion and the "effort of will involved in trying to alter the adjustment of the eyes". Closely related, more modern attempts to account for space constancy were mainly cancellation theories, in which the sensory effects of an eye movement are compensated by a simultaneous, equal and opposite extraretinal signal about the position of the eyes in the orbit (Sperry, 1950; von Holst \& Mittelstaedt, 1954). The retinal and extraretinal signals cancel each other in the brain, resulting in a space-constant representation of visual space. In these theories an oculomotor efference copy subtracts from the disturbing effects of a displaced retinal image following a saccade.

However, cancellation mechanisms alone presumably cannot achieve space constancy, since the extraretinal signals are not exact copies of the actual eye movement. First, their gain (ratio of extraretinal signal to actual eye movement) is usually less than one (Bridgeman \& Stark, 1991; Grüsser, Krizic, \& Weiss, 1987), so they are too small to afford complete compensation. Also dynamically, extraretinal signals of eye position are far from perfect. This results in large localization errors for flashed stimuli around the time of the saccade. Bischof and Kramer (1968) and Leonard Matin and colleagues (e.g., Matin, 1972) were among the first to study errors in the localization of flashed objects in the vicinity of saccadic eye movements. These and a large number of subsequent studies (e.g., Honda, 1989; Schlag \& Schlag-Rey, 1995) analysed the perception of short localized flashes before, during or after a saccade. The general finding was that these stimuli are systematically mislocalized. Mislocalization starts about $100 \mathrm{~ms}$ before the eyes begin to move, where flashes have a tendency to be seen as displaced in the direction of the saccade, and reaches a maximum around the time of the onset of the saccadic movement. These perceptual displacements are presumably a reflection of the sluggishness of the mechanism that compensates for the actual shift in retinal position brought about by the movement of the eye. Moreover, it has been recently demonstrated that the mislocalization of flashes before and during saccades is not spatially homogeneous. Ross, Morrone, and Burr (1997) showed that objects that are closer to the fixation than the saccade target are perceived as being displaced into the direction of the saccade, 
and those that are further away than the target are perceived as closer. In other words, targets flashed before and during saccades tend to converge towards the saccade target, which results in an apparent "compression" of the visual world around the saccade target.

Thus extraretinal information about eye position is notoriously imprecise, statically and dynamically. However, even a small error of the extraretinal signal should result in a disturbance of constancy. As a simple solution to this problem it has been proposed that the visual system has the built-in assumption that the world as a whole does not change during an eye movement, and that the remaining errors due to the imperfect cancellation mechanism are inhibited by a saccadic suppression mechanism (reviewed by Bridgeman, van der Heijden, \& Velichkovsky, 1994).

Saccadic suppression is a reduction of the visual sensitivity to events occurring before, during, and immediately after saccadic eye movements. Two separate types of saccadic suppression should be distinguished. First, there are many studies on the visual sensitivity to short flashes presented around the time of the saccade (for reviews see, e.g., Matin, 1974; Ross, Morrone, Goldberg, \& Burr, 2001). Typically, these studies have reported a moderate threshold elevation (two- to threefold) for detecting spots of light flashed briefly during saccades. Saccadic suppression is highest for low spatial frequencies; when gratings below 0.1 cycles/degree are viewed, it can be over a log-unit (Burr, Morrone, \& Ross, 1994). The second type of saccadic suppression, more relevant in the context discussed here, concerns the detection of image displacements that occur during saccadic eye movements. During fixation, the sensitive motion detectors of the visual system allow to perfectly perceive even very small displacements of visual objects. Due to the high retinal velocity during a saccade, however, these motion signals are basically "wiped-out" with each eye movement. This leads to a strong reduction in sensitivity (by three to four log units) for detecting displacements during saccades (e.g., Bridgeman, Hendry, \& Stark, 1975). Without direct evidence for a target jump from motion detectors, detection of intrasaccadic image displacement is dependent on the comparison of the egocentric pre- and postsaccadic target locations. Saccadic suppression of image displacement therefore seems to imply either that the required precise comparison is normally not performed, or that transsaccadic memory about the location of objects is not available to the visual system, or is very poor. Indeed, Bridgeman et al. (1994) in their theoretical account of visual stability proposed that (1) there is no need for a precise transsaccadic memory of object positions, (2) the spatial positions of objects are rather calculated anew after each saccade based on retinal information and efference copy signals, and (3), saccadic suppression "bridges the errors" that remain due to imperfect cancellation.

Recent experimental findings from my laboratory (Deubel, Bridgeman, \& Schneider, 1998; Deubel, Schneider, \& Bridgeman, 1996, 2002) have cast doubt on some of these assumptions, however. An important, but often neglected 
aspect of memory performance in general arises only at the moment when memory is probed, which is here when the saccade lands. Then, a comparison has to take place of the contents of transsaccadic memory and the actual reafferent visual information. The question arises how this comparison works and to what extent the stored information may be affected, and possibly, overwritten, by the new retinal information. I here propose that the effect of postsaccadic information on the use of transsaccadic memory is indeed an important factor for perceived visual stability. First evidence for this conjecture came from experiments on saccadic suppression of image displacement with simple targets (Deubel et al., 1996). These experiments demonstrated that saccadic suppression largely disappears with a stunningly simple manipulation, namely blanking the target with saccade onset and restoring it only 50-300 ms after the eyes stop at the end of a saccade-we called this effect the "blanking effect". The considerable accuracy with which subjects can judge transsaccadic displacements in the "blanking"' condition clearly requires both the maintenance of high-quality information about presaccadic target position across the saccade, and a precise extraretinal signal. Thus, it followed from our findings that precise information about the presaccadic target position and a precise extraretinal signal are indeed available for stimulus localizations after the saccade, but they ordinarily are not used in perception. We have suggested that this is because the visual system assumes, as a null hypothesis, the stability of any object that is continuously available both before and after the saccade. Only a very large discrepancy between eye movement magnitude and image position is able to break this assumption. This assumption is also broken, however, when the presaccadic object is not present immediately after the saccade. Only under these conditions are precise transsaccadic information and extraretinal signals used to achieve displacement detection. Because of its strong effect in unveiling information available transsaccadically, target blanking offers a tool for studying visual stability and the nature of spatial information transferred across the saccade.

While the absence of a postsaccadic target eliminates saccadic suppression of displacement, it turned out that its presence largely determines whether other stimuli in the field are seen as stable or as displaced across the saccade. This was demonstrated in experiments with two stimuli, a target and a distractor (Deubel et al., 1998). One of the manipulations in these experiments included a short intra- and postsaccadic blanking of one of the stimuli, while the other stimulus was displaced during the saccade. Even when the blank was very short (e.g., $50 \mathrm{~ms}$ ), the blanked object was invariably perceived as moving across the saccade, while the moved (but continuously present) object was perceived as stable. This was true whether the new reference object had originally been defined as the saccade goal or as the distractor. The fact that this striking illusion even occurred for object displacements of up to half of the size of the saccade illustrates that under these conditions perceptual stability is determined not by extraretinal signals but by the object that is found when the eyes land-this 
object serves as a spatial reference. The blanked object is then seen as displaced because its position is judged relative to the reference object, whose position is assumed to be stable.

Thus it seems that "landmark" objects found when the eyes land after a saccade are of fundamental importance for the transsaccadic localization of targets. The present investigation extends these previous findings by studying, in Experiment 1, the spatial range within which distractors become effective as transsaccadic references. For this purpose, landmark objects (pairs of small rectangles) were placed at various locations in the vicinity of the target. The landmark objects were present when the saccade landed, while the target was blanked for $200 \mathrm{~ms}$. The experiment analysed the effect of an intrasaccadic displacement of these distractors on the perception of the target displacement that occurred across the saccade. Experiment 2 investigated the extent to which information about the presaccadic spatial relations of objects contribute to postsaccadic localization. Experiment 3, finally, studied whether a highly salient visual frame is also effective in influencing perceived stability across saccades.

\section{EXPERIMENT 1}

\section{Method}

Participants. Seven paid subjects (six female, one male) participated in this experiment. They were naive with respect to the aim of the study, but were experienced with the equipment from other eye-movement related tasks, and had normal visual acuity. Subjects' age ranged from 20 to 32, with a mean age of 23 years. Each subject was run in three separate experimental blocks (see below).

Apparatus. Stimuli were presented on a 21 -inch video monitor at a frame rate of $100 \mathrm{~Hz}$. Screen background luminance was $2.2 \mathrm{~cd} / \mathrm{m}^{2}$; the luminance of the saccade target and of other stimuli was $25 \mathrm{~cd} / \mathrm{m}^{2}$. The subjects viewed the screen binocularly from a distance of $80 \mathrm{~cm}$. Head movements were restricted by a bite board and a forehead rest. Eye movements were measured with a SRI Generation 5.5 Purkinje-image eyetracker (Crane \& Steele, 1985) and sampled at a rate of $500 \mathrm{~Hz}$. Further details of computer control, calibration, and triggering of the saccade contingent display change are given in Deubel et al. (1996). The target consisted of a small white cross subtending a visual angle of $0.2^{\circ}$. The landmark object used in the experiment was composed of a pair two small white rectangles, $0.4^{\circ}$ wide $\times 0.56^{\circ}$ high, that appeared at various positions symmetrically above and below the horizontal meridian.

Procedure. Experiment 1 included three different experimental blocks. Each block contained 120 single trials and was repeated 10 times with each subject. The sequence of stimulus presentations for a typical trial is sketched in Figure 1. Initially, the subject maintained fixation on the target (small cross). 
After a random delay of 500-1200 ms, the target jumped left or right by $6^{\circ}$ to elicit a saccade. Simultaneously with the target jump, two small rectangles appeared symmetrically above and below the horizontal meridian. These distractors served as landmarks and were presented at various positions with respect to the target. The three different experimental blocks differed in the ranges of distractor positions with respect to the target. In a first block, the distractors always appeared at a vertical distance (DTv) of $0.47^{\circ}$ from the target. The horizontal distractor-target distance (DTh) was systematically varied. In the following, positive values of DTh will indicate distractor positions further eccentric than the target in the direction of the saccade; negative values will indicate distractors appearing between target and fixation and opposite to saccade direction as measured from the fixation position. The distractors could either appear at a location $3^{\circ}$ further eccentric to the target $\left(\mathrm{DTh}=+3^{\circ}\right)$, at the same horizontal position as the target $(\mathrm{DTh}=0)$, at a position in the middle between fixation and target $\left(\mathrm{DTh}=-3^{\circ}\right)$, at the position of the fixation $(\mathrm{DTh}=$ $-6^{\circ}$ ), and at a position $3^{\circ}$ opposite to the target location as measured from fixation $\left(\mathrm{DTh}=-9^{\circ}\right)$. Figure 1 displays an example for DTh $=-3^{\circ}$. A second block used distractor positions that were intended to provide a higher spatial resolution of the distractor effects around the target. For this purpose, the distractor-target distance was selected from DTh $=-2^{\circ},-1^{\circ},+1^{\circ}$, and $+2^{\circ}$, while DTv was kept constant at $0.47^{\circ}$. In a final experimental block, the vertical distance between target and distractors (DTv) was systematically varied, while the distractors always appeared horizontally aligned with the target (i.e., DTh $=$ $0)$. DTv was selected from $0.47^{\circ}, 1.47^{\circ}, 2.47^{\circ}, 3.47^{\circ}$, and $6.47^{\circ}$. The three different blocks were run in an order balanced across the subjects.

The primary saccade was elicited by the initial target jump. The computer detected a saccade when instantaneous eye velocity exceeded $30 \%$ s. Early triggering with the Purkinje-image tracker is essential because the tracker also records lens slippage within the eye, which leads to distortions in the measures of bulb rotation that are especially prominent late in the saccade (Deubel \& Bridgeman, 1995). At the time when the computer detected the saccade, the target was removed from the screen. Simultaneously, in two thirds of the trials, the distractors were displaced by $1^{\circ}$, either into the same or into the opposite direction as the primary saccade. Positive displacement values indicate shifts in the same direction; negative values indicate shifts in the direction opposite to the first saccade. In one third of the trials, the distractors remained stationary. Thus, the distractor displacements (DD) were $-1^{\circ}, 0^{\circ}$, or $1^{\circ}$. Since these small displacements occurred during the saccade, the subjects never noticed them. $200 \mathrm{~ms}$ after saccade onset (i.e., about $160 \mathrm{~ms}$ after saccade end) the target was presented again. It reappeared at various horizontal offsets from the presaccadic target location. The size of this second target displacement (TD) was $1^{\circ}$ or $0.4^{\circ}$, either into the same or into the opposite direction of the first saccade. At the end of each trial, in a two-alternative forced-choice procedure, the 


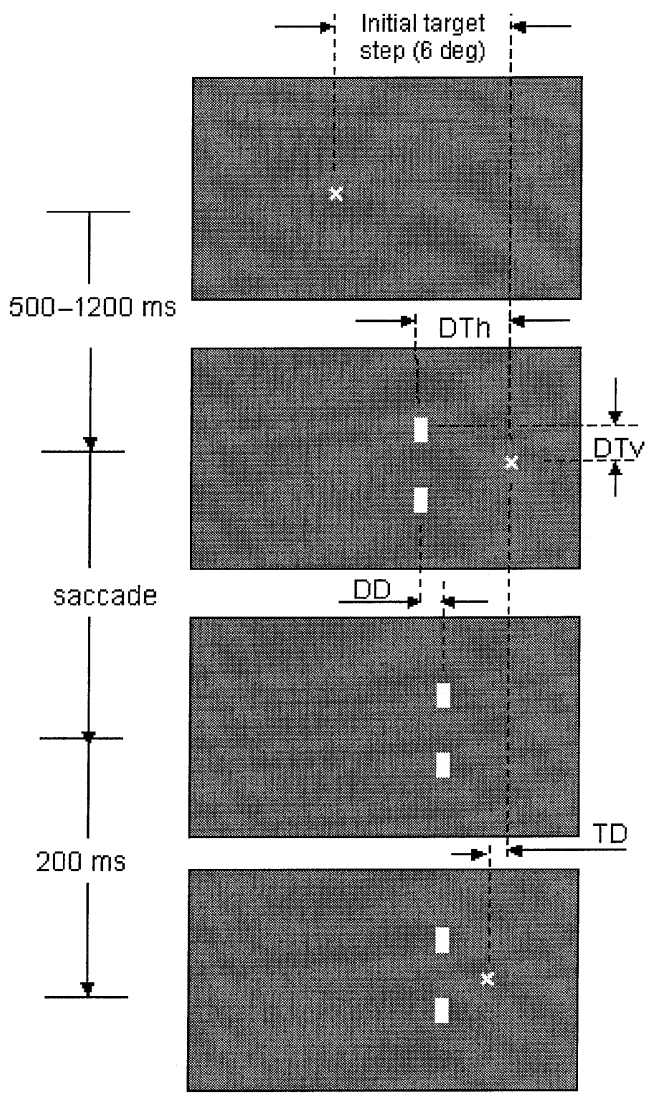

Figure 1. Stimulus sequence for a typical trial from Experiment 1. Initially, the subject maintained fixation on the target (small cross). After a random delay, the target jumped right or left by $6^{\circ}$ to elicit a saccade. Simultaneously with the target jump, two small rectangles appeared symmetrically above and below the horizontal meridian, at a vertical distractor-target distance (DTv). The horizontal distractor-target distance (DTh) was systematically varied; the figure provides an example for DTh $=$ $-3^{\circ}$ (positive displacement values indicate displacements into the same direction as the primary saccade). Triggered by the saccade, the target disappeared. The distractors remained continuously visible, but were displaced horizontally; the distractor displacement (DD) was $-1^{\circ}, 0$, or $+1^{\circ}$. The target reappeared $200 \mathrm{~ms}$ after saccade onset at a displaced position. The size of this second target displacement (TD) was $1^{\circ}$ or $0.4^{\circ}$, either in the same or in the opposite direction as the first saccade. The final target location served as the starting position for the next trial.

subject's task was to report the direction of the second target shift with respect to the direction of the primary saccade ("forward" vs. "backward"), while ignoring the distractors. The final target location served as the starting position for the next trial. 
Data analysis. The experimental data were stored on disk for off-line analysis. To determine gaze direction, an off-line program searched the eye record every $2 \mathrm{~ms}$ for the beginning of the saccade movement and for the end of the overshoot after the saccade (Deubel \& Bridgeman, 1995) and then calculated starting and end points of the saccades.

The main goal of the analysis of the perceptual data was to gain a quantitative measure of the effect of the distractor displacement on perceived target shift. For this purpose, psychometric functions relating the judgements to the effective target displacements were determined for each distractor condition (as examples, see graphs a and c in Figure 2). The next analysis step was to compute the amount of target mislocalization that was induced by the distractor manipulation. Target mislocalization can be estimated as the value of target displacement where the subject sees $50 \%$ forward displacements. For this purpose, a bootstrap procedure $^{1}$ was applied to each psychometric function, which fitted, by weighted linear regression, a cumulative Gaussian function to the set of data points and then computed the threshold (for a criterion level of 50\%). Then these values were plotted as a function of distractor displacements for all subjects (see graphs $b$ and $d$ in Figure 2). Finally, a linear regression across the subjects provided a measure of the effectiveness of the distractors. Further account of the data analysis is given in the following section.

\section{Results}

Perceptual displacement judgements. To illustrate the typical data pattern revealed from Experiment 1, Figure 2 provides examples of the experimental results for two landmark locations, namely for DTh $=0$ (Figure 2, left graphs) and for DTh $=-1^{\circ}$ (Figure 2, right graphs). The data points in Figure 2a display the relative frequencies of a typical subject indicating a perceived "forward" displacement of the target, as a function of the effective target displacement, for $\mathrm{DTh}=0$, i.e., where the presaccadic distractors appeared directly above and below the target. The data are shown separately for the different intrasaccadic distractor displacements (DD). The magnitude of the effect of distractor displacement on target localization can be estimated by examining the deviation of the curves for leftward and for rightward displacement of the distractors where they cross the 50\% "neutral" position (horizontal lines in Figures 2a and 2c). These values indicate the mislocalization of the target in the various

\footnotetext{
${ }^{1}$ This " $\mathrm{C}$ ” program is freely available from http://www.cs.ualberta.ca/ wfb/software.html. It fits, by weighted linear regression, a cumulative Gaussian psychometric function to a set of binary data. It then computes the threshold (for a given criterion level of performance) and the gradient. The bootstrap procedure is similar to that given in Foster and Bischof (1991), but a more robust procedure has been adopted in that standard deviations are computed from centiles, assuming a normal distribution.
} 
(a)

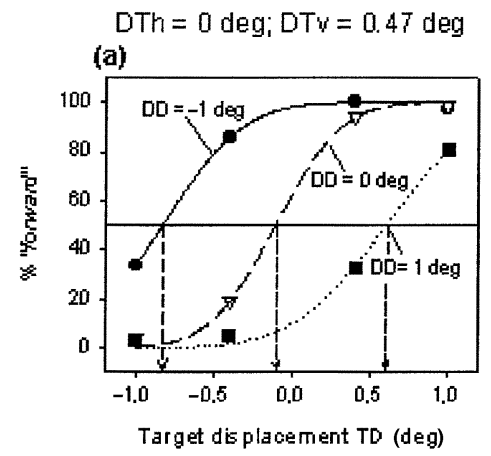

(b)

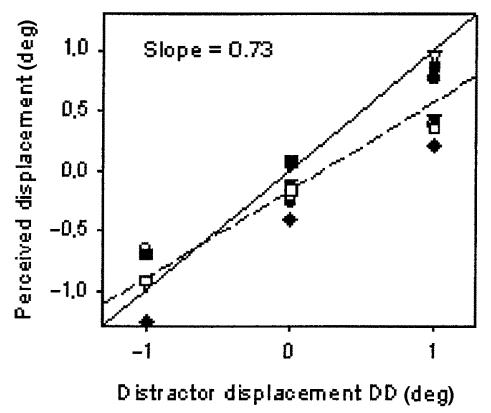

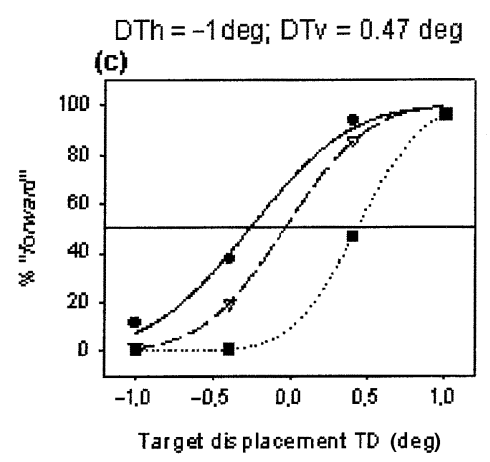

(d)

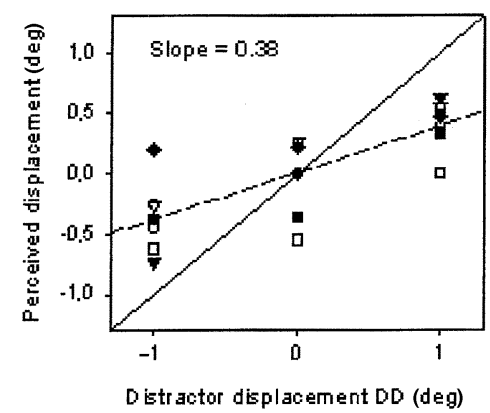

Figure 2. Upper graphs: Psychometric functions relating the percentage of "forward" judgements to the effective target displacement as a function of the effective target displacement, for DTh $=0$ (Figure 2a) and DTh $=-1^{\circ}$ (Figure 2c). Parameter is the size and direction of the intrasaccadic distractor displacement (DD). The data points are fitted with a cumulative Gaussian. The displacement values where the curves cross the horizontal lines plotted in Figures 2a and 2c indicate the $50 \%$ "neutral" position, i.e., the target displacement where it is perceived as stable. In other words, this is amount of target shift that is necessary to compensate for the effect of the distractor displacement. The lower diagrams in Figure 2 (Figures $2 b$ and 2d) display these induced mislocalization values resulting from the previously described analysis as a function of distractor displacement DD, for all seven subjects. The slope of the regression line is a direct measure for the effectiveness of the distractors, it is 0.73 for DTh $=0$ and 0.38 for DTh $=-1^{\circ}$.

conditions. In order to compute these values, which are indicated in Figure $2 \mathrm{a}$ by the vertical dashed arrows, each psychometric function was fitted separately with a cumulative Gaussian. The curves in the plot show the results of the data fitting. Then, the $50 \%$ point was determined, i.e., the actual target displacement where the subject would perceive a stable target. This is the target displacement necessary to perfectly compensate for the effect of the distractor shift on displacement detection.

For DD $=0$, i.e., without intrasaccadic distractor shift, the resulting psychometric function (dashed curve) indicates that the subject has an almost 
veridical perception of target displacement, since the perceived target displacement is very close to 0 . Also, the psychometric curve is quite steep, indicating that the variability of the judgements is low; in other words, the subject detects the displacements with considerable accuracy. For the remaining cases including a $1^{\circ}$ intrasaccadic distractor shift, however, the psychometric functions are shifted to the left or to the right, depending on the direction of the distractor displacement (continuous and dotted curves). This indicates that the subject now experiences a quite dramatic illusion: In order to be perceived as stable, the target now has to move by about $0.7^{\circ}$, in the same direction as the distractor shift! This demonstrates that the position where the landmark is located after the end of the saccade has a decisive influence on where the target is expected to reappear after the saccade, inducing a shift of the expected target location in the direction of distractor displacement.

Figure $2 \mathrm{~b}$ displays the induced target mislocalization as computed in the previously described analysis as a function of distractor displacement, for all seven subjects. In order to quantify the strength of the distractor-induced effect further, a linear regression was calculated. The slope of the regression line is a direct measure the effectiveness of the distractors, describing the extent to which a certain distractor displacement results in a perceived target displacement. It can be seen that the slope of the regression line for this experimental condition $\left(\mathrm{DTh}=0, \mathrm{DTv}=0.47^{\circ}\right)$ is 0.73 , indicating that a distractor displacement of $1^{\circ}$ will lead to an induced target mislocalization of $0.73^{\circ}$.

The graphs on the right provide another example for a similar data analysis, but now for DTh $=-1^{\circ}$, i.e., for the experimental condition where the (presaccadic) distractors were located $1^{\circ}$ closer to the fovea from the target. It can be seen from the clear separation of the psychometric functions in Figure $2 c$ that the distractors still have a substantial effect on transsaccadic localization. However, the regression analysis (Figure 2d) now yields a considerably smaller slope of 0.38 , indicating that distractor strength is only half of the value for DTh $=0$. This suggests that the magnitude of interaction between distractors and target decreases rapidly with increasing target-distractor distance.

Figure 3 summarizes the perceptual data for all conditions, showing the slope values gained from the regression analysis for the variation of the horizontal distractor positions Figure 3a, filled circles) and for the variations of the vertical target-distractor distance (Figure 3b, open squares). As expected, distractor efficiency is highest $(0.73)$ when the distractors are closest to the target position, i.e., for DTh $=0$ and DTv $=0.47$. For larger horizontal target-distractor distances, distractor efficiency drops quickly (filled circles). So, if the presaccadic distractors appear in the middle between fixation and target (i.e., for DTh $=$ $-3^{\circ}$ ), efficiency has dropped to only 0.14 . Objects at the fixation $\left(\mathrm{DTh}=-6^{\circ}\right.$ ) seem to exert only a negligible effect on localization, and the same holds for objects in the contralateral visual field $\left(\mathrm{DTh}=-9^{\circ}\right)$. When the vertical distractor-target distance increases while the distractors are located above and 


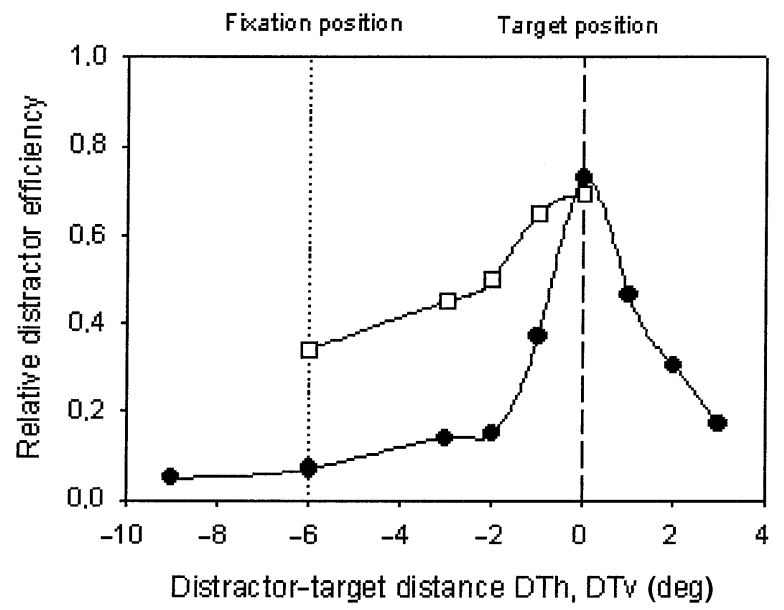

Figure 3. (a) Distractor efficiency gained from the regression analysis for the various horizontal distractor-target distances $\left(\mathrm{DTv}=0.47^{\circ}\right)$. The vertical dotted line indicates the fixation position, the vertical dashed line indicates target position. (b) Distractor efficiency as a function of vertical distractor-target distances $\left(\mathrm{DTh}=0^{\circ}\right)$.

below the target $(\mathrm{DTh}=0$ ), distractor efficiency also drops (Figure $3 \mathrm{~b}$, open squares). However, interestingly, the decrease is considerably shallower than for the variable horizontal positions. Even when the distractor elements are located $6^{\circ}$ above and below the target, they are sill amazingly effective in target localization - an intrasaccadic distractor displacement of $1^{\circ}$ still leads to a target mislocalization of $0.34^{\circ}$.

The presented results allow more precise specification of the distractor effects on transsaccadic localization. Distractors are highly effective as long as they appear close to the target. If under these conditions the target returns only after a gap, it is the distractor displacement that largely determines where the target is expected to reappear. The attraction exerted by the distractors decreases quickly when the distractors appear further away in the horizontal direction, implying that the distractor effect is spatially very selective. However, somewhat unexpectedly, objects with a large vertical distance from the target are still very effective when they align horizontally with the target, for horizontal saccades. This may occur because there are always two targets centred vertically above and below the target, so that the mean vertical position of the targets coincides with the target level. It is important to note that the distractor displacement did not eliminate the perceptual advantage of blanking; it merely biased the judgements of displacement. This becomes obvious from the steep psychophysical functions similar to those that have characterized the blanking effect in the previous experiments (Deubel et al., 1996, 1998). Taken together, 
the results imply that target location is evaluated with reference to the continuously visible distractors when the target is blanked after the saccade.

Oculomotor behaviour. Mean latencies of the primary saccades to the target are shown in Figure 4a as a function of the horizontal distractor-target distance (DTh). Error bars indicate standard error. Latencies of primary saccades are fast and show a unimodal distribution (data not shown). As known from the "remote distractor effect"' (Walker, Deubel, Schneider, \& Findlay, 1997), saccades tend to be markedly slower if the target is presented together with a distractor at fixation or in the opposite hemifield, which is reflected in Figure 4a for DTh $=$ $-6^{\circ}$ and DTh $=-9^{\circ}$. The primary saccades are generally directed to the target except for the cases where the distractors appear closely before of behind the target. In these cases, a marked averaging effect is present with the saccades landing at an intermediate location (data not shown).

An interesting question concerns the behaviour of the oculomotor system after the initial saccade. Given that perceptual localization of the target depends on the distractor displacement, the question arises whether corrective saccades occur that are triggered before the target is presented again, and whether these would be directed to the distractors or to the expected target location. Therefore, I also analysed corrective saccade latencies and amplitudes. A latency histogram for secondary saccades is presented in Figure $4 \mathrm{~b}$, showing the data only for all those cases where the distractors were more than $1^{\circ}$ away from the target. The vertical dashed line in Figure $4 \mathrm{~b}$ indicates the time of target reappearance. The large majority of the secondary saccades occur with a latency of more than $300 \mathrm{~ms}$ after the primary saccade, and are therefore obviously triggered by target onset. Closer analysis of their amplitudes not presented here demonstrates that they are indeed directed to the target without effect of distractor location. This suggests that the secondary saccades largely ignore the distractors and wait for the target to reappear. However, a small proportion of corrective saccades with latencies shorter than $300 \mathrm{~ms}$ exists for the critical case where the distractors were close to the target $(\mathrm{DTh}=0)$ (see Figure $4 \mathrm{c})$. These corrective saccades are obviously not triggered by the target onset but are programmed before target reappearance. Amplitude analysis of these saccades demonstrates that they are indeed directed to the locations indicated by the distractors.

\section{EXPERIMENT 2}

The previous experiment demonstrates that visual landmarks are highly efficient determinants for the perception of target displacements across saccadic eye movements. An intrasaccadic shift of distractors located close to the saccade target leads to a perceived target displacement more than $70 \%$ of the distractor movement, suggesting that the presaccadic spatial relation between target and 

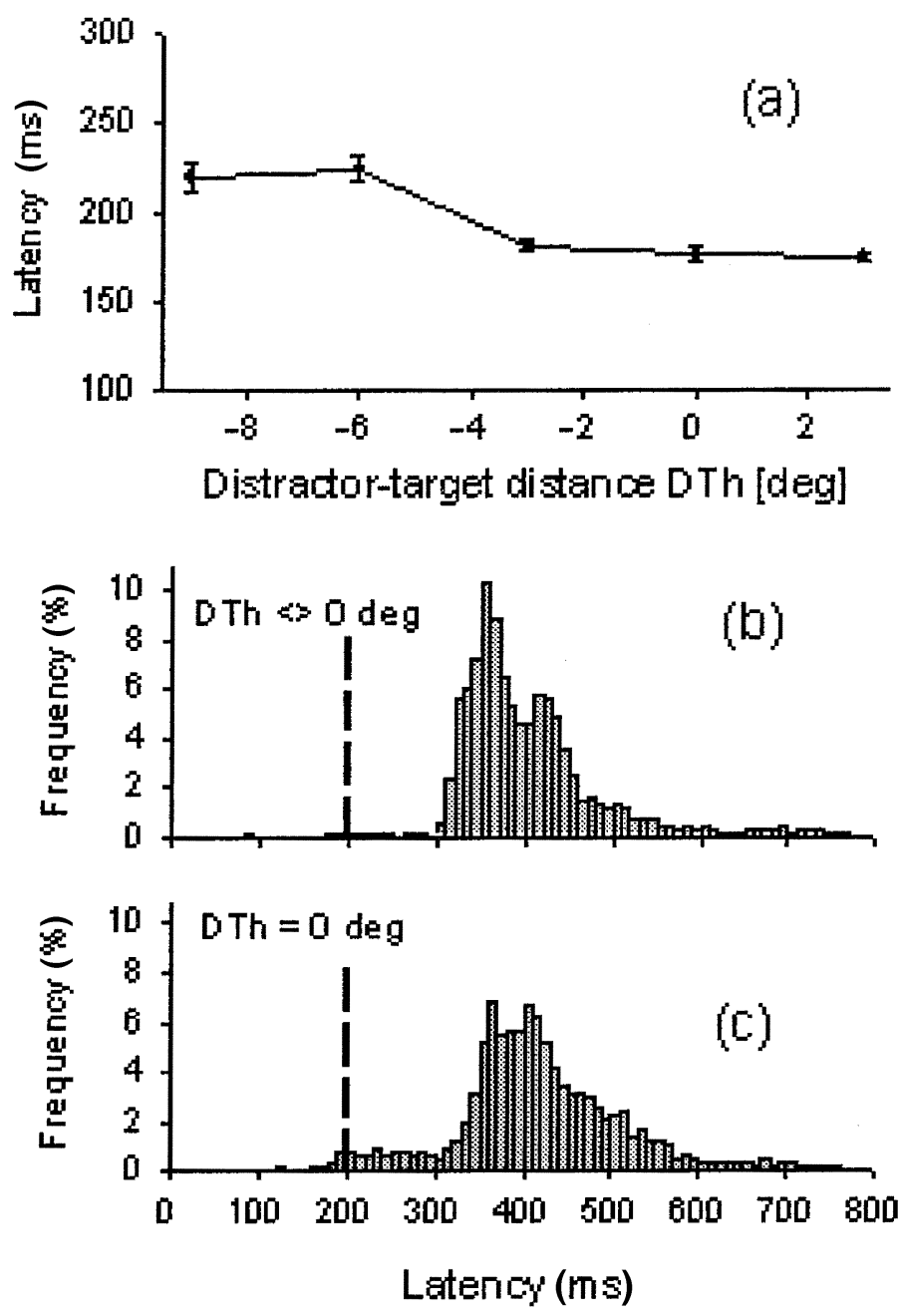

Figure 4. (a) Mean latencies of the primary saccades as a function of the distractor position DTh. Error bars indicate standard error. (b) Latency histogram for secondary saccades, showing the data only for all cases where the distractors are more than $1^{\circ}$ away from the target. The vertical line indicates the time of the reappearance of the distractor. (c) Latency histogram for secondary saccades, for DTh $=0$ and DTv $=0.47^{\circ}$.

distractors is stored across the saccade and serves to generate an expectation of target location after the saccade.

However, the data also allow for an alternative interpretation, at least in the conditions where the horizontal offset between distractors and target was small 
or zero. Instead of using the relational information of target and distractors from before the saccade, the subject may under these conditions simply expect, by default, the target after the saccade to appear at the (horizontal) location of the landmark. This would lead to a similar mislocalization tendency as found in the experimental data. A second puzzling finding of the previous experiment is the great efficiency of distractors even with large vertical distances from the target. Possibly, a critical feature in this experimental condition is that the distractors were presaccadically aligned with the target. In order to investigate these two questions, an experiment was performed where the horizontal distractor locations were varied such that they were no longer aligned with the target location. Also, the distance between distractors and target in the vertical direction was systematically varied. In order to reduce the number of experimental conditions, the target was never shifted in Experiment 2. Nevertheless, all subjects were sure to perceive clear target displacements on most of the trials, demonstrating the strength of the illusion.

\section{Method}

Participants and apparatus. Five paid subjects participated in this experiment. Each subject was run 10 times in the experimental block consisting of 160 single trials. The experimental apparatus and the target and distractors were the same as in the previous experiment.

Procedure. The initial part of the stimulus sequence in Experiment 2 was similar to Experiment 1 . The subject initially fixated the fixation cross, then the target jumped left or right by $6^{\circ}$ to elicit a saccade. Again, simultaneously with the target jump, two small rectangles appeared symmetrically above and below the horizontal meridian that served as a visual landmark. The vertical distractor-target distance (DTv) was systematically varied between $0.47^{\circ}, 1.47^{\circ}, 3.47^{\circ}$, and $6.47^{\circ}$. The distractors were now presented with a horizontal offset DTh of $-1.5^{\circ},-0.5^{\circ},+0.5^{\circ}$, or $+1.5^{\circ}$ with respect to the target. Triggered by the primary saccade, the target disappeared from the screen while the continuously visible distractors were displaced. Distractor displacement (DD) was $-1^{\circ}, 0^{\circ}$, or $+1^{\circ}$. Not all combinations of DTh and DD were applied; the conditions DTh $=-1.5^{\circ}$, DD $=-1^{\circ}$, and $\mathrm{DTh}=+1.5^{\circ}$, DD $=+1^{\circ}$ were not presented (see Figure 5 for further illustration). Note that the spacing of target and distractors and the jump sizes are selected such that neither the presaccadic nor the postsaccadic distractor positions were ever aligned with the target. The target reappeared after a blanking period of $200 \mathrm{~ms}$, always at the same spatial position as it was located before the saccade. Again, at the end of each trial the subject's task 

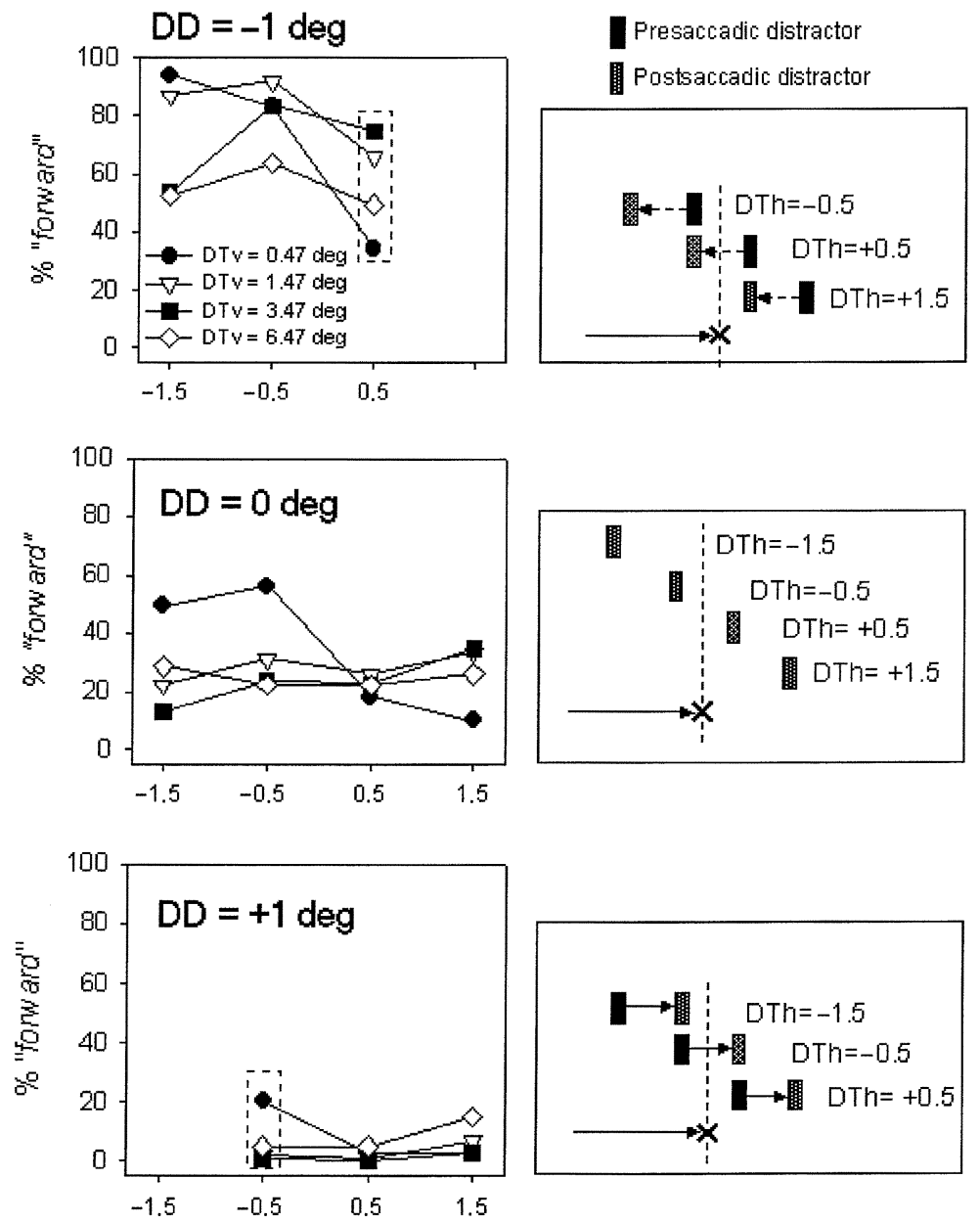

Postsaccadic distractor / target distance

Figure 5. Displacement judgements and illustration of the pre- and postsaccadic distractor positions with respect to the target (cross) in Experiment 2. Data and illustrations are presented separately for the three distractor displacements DD of $-1^{\circ}, 0$, and $+1^{\circ}$. Each graph shows the percentage of "forward" judgements as a function of the postsaccadic horizontal distance between landmark and target. The separate curves show the data for the four different values of DTv.

was to report the direction of the perceived second shift of the target (note however that the target did not move during the saccade) with respect to the direction of the primary saccade ("forward" vs. "backward"). The final target location served as the starting position for the next trial. 


\section{Results}

The perceptual judgements are given in Figure 5, together with an illustration of the pre- and postsaccadic distractor positions with respect to the target location (cross). Data and illustrations are presented separately for the three distractor displacements DD of $-1^{\circ}, 0$, and $+1^{\circ}$. Each graph shows the percentage of "forward" judgements as a function of the postsaccadic distance between distractors and target. Again, positions of distractors that appear closer to the initial fixation are designated as negative values. The separate curves show the data for the four different values of DTv.

The central question of this experiment was whether perceptual judgement of target displacement is mainly dependent on direction and size of transsaccadic distractor displacement or rather on the relative postsaccadic position where the target reappeared with respect to the distractors. The data in Figure 5 provide a clear answer. If perceptual localization depended only on the postsaccadic position of the target relative to the landmark, subjects should see "forward" displacements for negative postsaccadic distractor-target distances (i.e., for the cases where the target appeared ahead of the distractors), and "backward" displacements for positive distractor-target distances. Clearly this is not the case; the curves tend to be flat for almost all values of DD and DTv. Rather, the major effect occurs as a function of distractor displacement DD: for negative values of DD, subjects perceive mainly "forward" displacements (upper plot), while for positive values of DD, subjects invariably perceive "backward" displacements (lower plot). Particularly informative in this context are the cases where both sources of information are in conflict, for example for the combinations $\mathrm{DD}=-1^{\circ}, \mathrm{DTh}=+1.5$ (upper graph) and for $\mathrm{DD}=+1^{\circ}, \mathrm{DTh}=-1.5^{\circ}$ (lower graph). The corresponding data are highlighted in the graphs by the dashed rectangular frames. In the first case, a judgement purely based on relative position in the postsaccadic display would predict a "backward" judgement bias, since the distractors remain ahead of the target after the saccade. A judgement determined by the direction and size of the distractor displacement would yield a "forward" bias, however, since the distractors have moved backwards. The second case presents the mirror-symmetrical situation; here a judgement based on distractor position would predict a "backward" judgement bias while a judgement determined by distractor displacement would yield a "forward" bias. The respective data from Figure 5 clearly demonstrate that the winner that determines perceptual performance is intrasaccadic distractor displacement rather than relative postsaccadic distractor position.

This conclusion is confirmed by further statistical analyses. The arcsinetransformed probability values were analysed in a three-way ANOVA (DD * DTh * DTv). The analysis yielded a highly significant main effect of the distractor displacement DD, $F(2,8)=275.2, p<.001$, and nonsignificant main effects of DTh, $F(3,12)=2.47, p>.1$, and DTv, $F(3,12)=0.75, p>.1$. As 
expected, the analysis also revealed significant interactions DD $\times \mathrm{DTh}, F(6,24)$ $=3.7, p<.01$, and DD $\times \mathrm{DTv}, F(6,24)=4.37, p<.001$, indicating that the effect of DD on perceived displacement also depends on the other variables.

\section{EXPERIMENT 3}

The previous experiments concerned the effects of small, well-localized landmark objects on the perceived displacement of a target across the saccade. The findings revealed that these landmarks are highly efficient determinants of transsaccadic localization, specifically, if they are located close to the target. Moreover, the second experiment showed that the visual system considers relational information of target and distractors stored across the saccade for the perceptual displacement judgement. The question arises whether other contextual information also contributes to the judgement. Most prominently, in most experiments the stimuli are presented on a monitor screen providing a well visible stable frame. This stable frame could potentially contribute to the perceived visual stability across saccades, leading to smaller effects in Experiments 1 and 2 than one might expect in complete darkness or with a perfectly uniform background. Therefore, the third experiment analysed quantitatively the effect of a large, highly salient frame on transsaccadic displacement detection.

\section{Method}

Participants and apparatus. Five paid subjects participated in this experiment. Each subject was run five times in the experimental block consisting of 144 single trials. Experimental apparatus and the target were the same as in the previous experiments. Instead of presenting two small, localized distractors a large area of the display was now filled with a black frame that left a grey rectangular area of $14^{\circ} \times 6^{\circ}$, centred on the screen (Figure 6a).

Procedure. The initial part of the stimulus sequence in Experiment 3 was the same as in the previous experiments. The subject initially fixated the target, then it jumped left or right by $6^{\circ}$ to elicit a saccade. Triggered by the primary saccade, the target disappeared from the screen. At the same time, the continuously visible frame was displaced by either $-0.75^{\circ}, 0^{\circ}$, or $+0.75^{\circ}$. Since this displacement took place during the saccade, the subjects never noticed it. The target was presented again after a blanking period of $200 \mathrm{~ms}$. It reappeared at various horizontal offsets from the presaccadic target location. The size of this second target displacement was $1^{\circ}$ or $0.4^{\circ}$, either in the same or in the opposite direction as the first saccade. At the end of each trial the subject reported the direction of the second target shift with respect to the direction of the primary saccade ("forward" vs. "backward"). The final target location served as the starting position for the next trial. 

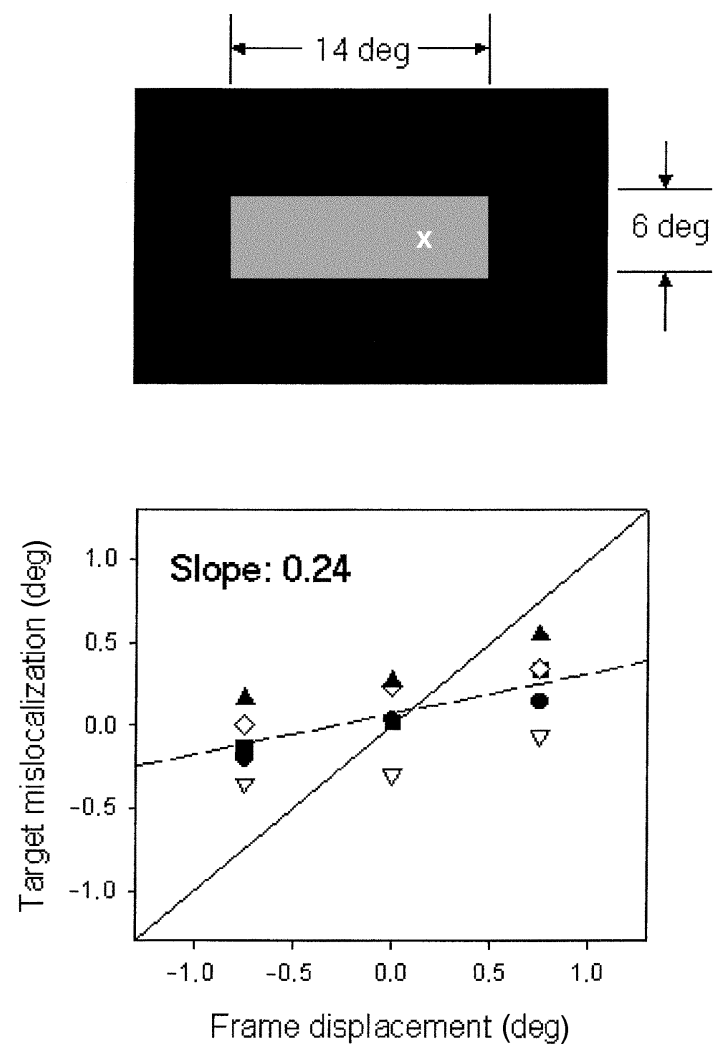

Figure 6. (a) Stimuli in Experiment 3. A large area of the display was filled with a black frame that left a grey rectangular area of $14^{\circ} \times 6^{\circ}$, centred on the screen. (b) The data points indicate the induced target mislocalization for three values of frame displacement, separately for each of the five subjects. The slope of the regression line estimates the relative efficiency of the frame as a spatial landmark to be 0.24 .

\section{Results}

In order to obtain a quantitative measure of the effect of the displacement of the frame on perceived target shift, the same analysis as in Experiment 1 was performed. From the psychometric functions relating the percentage of "forward" judgements to the effective target displacement, induced target mislocalization was estimated for each of the three values of distractor displacement and for each subject. These values are shown in Figure $6 \mathrm{~b}$ as a function of the frame displacement. It can be seen that frame displacement indeed has a systematic effect on perceived target displacement. The slope of the regression line also shown in the plot estimates the efficiency of the frame as a spatial landmark 
as 0.24 . In other words, an intrasaccadic frame displacement of $1^{\circ}$ induces a target mislocalization of only $0.24^{\circ}$. This is an amazingly weak effect given the fact that small distractors such as those provided in Experiment 1 can yield considerably larger effects even if they appear with a (vertical) distance of $6^{\circ}$ with respect to the target.

\section{DISCUSSION}

\section{Postsaccadic landmarks are important determinants of object localization and visual stability across saccades}

This study represents a continuation of the earlier work on the blanking effect. The central result of the previous investigations was that when a saccade target is blanked even for a short interval during and after a saccade, its transsaccadic displacement becomes much more visible than when the target is continuously present (Deubel et al., 1996). A second important finding was that the object that is found by the visual system immediately after the saccade is normally perceived as stable, and it is taken as a spatial reference for judging whether other (blanked) objects had moved (Deubel et al., 1998, 2002). The central aim of the three experiments presented here was to study in detail the possible role of distractor objects present before and after the saccade as landmarks for the transsaccadic localization process. The basic experimental manipulation consisted in a systematic displacement of these landmarks during the saccade. The relative effectiveness of the presence and the displacement of the landmarks was determined by analysing to what degree they modified the perceived shift of a small saccade target that was blanked for $200 \mathrm{~ms}$ during and after the saccade. While the first and the second experiment looked at the effect of small, localized distractor objects, the third experiment studied the effect of a high-contrast, massive frame on transsaccadic localization.

All three experiments revealed strong effects of the landmark objects. Though subjects reported to have never perceived the intrasaccadic displacement of the distractors, the displacement had a consistent effect on perceived target jumps. The first experiment varied, in three experimental blocks, the distractor locations over a wide range of spatial positions. It turns out that the effect of landmarks is spatially selective. It is highest when the distractors appear close to the target, and when landmark objects and target are aligned horizontally. Under these conditions, the landmarks indeed largely determine transsaccadic localization: More than $70 \%$ of a distractor displacement is reflected in the induced target mislocalization. This implies that under normal perceptual conditions the efference copy signal of eye position after the saccade plays only a minor role in transsaccadic localization; rather, transsaccadic displacement judgements and perceived visual stability are based on the evaluation 
of postsaccadic landmark objects. The importance of visual reference frames is also supported by earlier experiments, which indicated that in normal visual scenes, visual information dominates over eye position information in judgements of visual direction, when both signals are in conflict (Matin, Picoult, Stevens, Edwards, Young, \& MacArthur, 1982; Stark \& Bridgeman, 1983).

The results show that the spatial layout found by the visual system after the saccade forms a major source of information used to establish space constancy. Found objects serve as anchor points to determine the expectations where objects such as the blanked target should appear after a saccade. This is in line with what was found in our former investigation (Deubel et al., 1998). When, in these experiments, one of two objects presented before the saccade is blanked with saccade onset, then only the other object is available to become the reference object. Since the system is not particularly selective about visual features, this distractor becomes the reference object by default, provided that the location of the distractor is sufficiently close to the saccade goal object. By the time the blanked target reappears, the system is already committed to the other object as the reference object. The blanked target is then seen as displaced because its position is judged relative to the reference object, whose position is assumed to be stable.

Further evidence provided in Deubel et al. (1998) suggests that not just single, isolated objects, but also spatially extended, visually more complex structures may serve as landmarks. In their third experiment, they presented a target embedded in an extended background structure consisting of circles and ellipses. When this background was shifted during the saccade, the target reappearing after a blank was mislocalized by about $50 \%$ of the background shift.

Another important contribution emphasizing the role of landmarks for spatial localization has been provided by Germeys, De Graef, Panis, van Eccelpoel, \& Verfaillie, (2004). These authors studied how well the locations of bystander objects, i.e., objects that are not the target of the saccade, are remembered across saccadic eye movements. In line with the findings presented here, their results clearly demonstrate that the transsaccadic memory of the bystander locations is largely determined by the configurational information of the stimulus layout maintained across the saccade. Moreover, their results show that the task relevance of the various display items determines whether and how they are used for transsaccadic localization.

\section{The effect of landmarks is limited to a spatial range close to the target}

Experiment 1 demonstrates that the efficiency of the landmarks drops steeply for distractors eccentric to the target. The data from Figure 3 show that the distractor effect largely disappears beyond a horizontal range of $3^{\circ}$ from the target. This 
also implies that the visual system does not consider stimuli at the fixation location as spatial landmarks. However, it should be pointed out that in the present study the size of the region around the saccadic target where distractors are relevant for localization has been measured for only one saccadic amplitude, which was $6^{\circ}$, and may not generalise to all saccades. Indeed it is likely that the spatial extent of the region may scale with saccadic size, as does saccadic suppression of displacement (e.g., Bridgeman et al., 1975).

Another surprising finding of the present study is that a visually dominant frame, as in Experiment 3, when displaced during the saccade, has much less effect on localization than a rather small pair of isolated distractors. This seems to suggest that the postsaccadic localization process treats the frame as a nearly irrelevant background structure. Alternatively, the effect of the rectangular frame may be determined solely by the horizontal distance between the target and the closest vertical edge of the frame rectangle. Under this assumption, frame efficiency can be estimated from the data of Experiment 1, which would indeed lead to a predicted efficiency below 0.2 .

There is further empirical evidence that supports the assumption of a preferential transsaccadic processing of the saccade target. Bischof and Kramer (1968), for instance, found perceived locations to be corrected more quickly near the saccadic goal than at other retinal positions. In a saccadic suppression experiment in which either the saccade target or another visual object such as the previous fixation target moved during the saccade, Heywood and Churcher (1981) showed that subjects often misattribute an intrasaccadic displacement of the saccade goal to a displacement of the other object, tending to preserve space constancy preferentially for the saccade goal. In a study by Irwin (1992), subjects were presented an array of letters while they fixated a central fixation point; the onset of a peripheral stimulus indicated the saccade target. The letters disappeared with the onset of the saccade; the subjects were required to report one of the letters in a partial report paradigm. Irwin found that subjects could remember only three or four letters, and that report of the letters near the saccade target (never foveated) was much more accurate than of the other letters in the array. This suggests that information near the saccade target is more likely to be encoded in transsaccadic memory than information from more distant locations.

McConkie and Currie (1996) used full-color pictures of natural scenes that changed during the saccade. In their Experiment 2, the scene expanded or contracted during the eye movement. McConkie and Currie found that the detectability of these image changes was a direct function of the displacement size at the location where the eyes landed, confirming the importance of the local region around the saccade target. Currie, McConkie, Carlson-Radvansky, and Irwin (2000), also using full-colour pictures of natural scenes, studied the detectability of intrasaccadic displacements of objects in the display. They found that displacements of the saccade target object were much easier to detect than displacements of the background (with the saccade target object 
remaining stationary). Finally, an important role of the saccade target has been suggested by Ross et al. (1997), demonstrating that stimuli flashed shortly before a saccade are mislocalized such that they are perceived closer to the saccade target.

However, it should be noted that the preferential processing of the target also seems to depend, in a yet unknown manner, on the specific task. Verfaillie and De Graf (2000), for example, had subjects saccade from one biological motion walker at the fixation position to another in the visual periphery. During the saccade, either the walker at the launch site or the walker which was the saccade target changed in depth orientation or in location. The results show that change detection for the walker at the saccade target was not more accurate than for the walker at the presaccadic fixation. The walker targets were large, however, extending to eccentricities peripheral enough to have little effect on localization in the current experiments.

The fact that distractors close to the target are preferentially considered in the spatial localization process is probably related to an attentional component of saccade processing and the anchoring mechanism. A number of investigators have shown that attention movements obligatorily precede saccadic eye movements, leading to a selective improvement of the detection and identification of items presented at the saccade target location, and to a deterioration of performance at other stimulus locations (e.g., Deubel \& Schneider, 1996; Hoffman \& Subramaniam, 1995; Kowler, Anderson, Dosher, \& Blaser, 1995; Schneider \& Deubel, 2002). This is so even if subjects are instructed to attend to a location other than the saccade target. To demonstrate this, Irwin and Gordon (1998) manipulated attention in a transsaccadic letter recognition task. Subjects were encouraged to attend to one region in a display while they moved their eyes either to the region they were attending or to another region. The results show that accuracy was high at positions that subjects were asked to attend to, but it was about equally high for positions close to the saccade target, even if the subjects were asked to attend elsewhere. Whether landmark objects that are never attended can also affect transsaccadic localization is on open empirical question, however.

\section{For horizontal saccades, postsaccadic perceptual anchoring is based on vertically oriented structures}

Visual objects that are vertically aligned with the target have a strong effect on localization even when they appear at a distance of up to $6^{\circ}$ (Experiment 1$)$. This initially unexpected finding was qualified in Experiment 2 showing that perfect (presaccadic) alignment of target and landmark is not necessary, but that the visual system tolerates horizontal displacements of at least a degree. The question nevertheless arises why objects that that can be structured into a ver- 
tically oriented Gestalt are so effective as landmarks for horizontal saccades. A possible answer results from the assumed function of these landmarks for visual stability and spatial recalibration across saccades. For saccades in a complex visual environment, only the spatial components oriented orthogonal to the saccade direction are useful for a calibration of errors resulting from, e.g., an improper gain or dynamics of the extraretinal signal. For horizontal saccades this implies that vertically oriented bars, luminance edges, etc. are the major sources of spatial localization across the saccade.

\section{Transsaccadic localization is dependent on relational information from before the saccade}

An important question addressed in Experiment 2 of the present paper was whether the visual localization system would use the relational information of target and distractors from before the saccade, stored across the saccade, to generate an expectation of the postsaccadic target location. Alternatively, the visual system may simply tend to take the position of any localized object found after the saccade as the assumed target location. Some evidence for the latter assumptions came from a recent experiment studying the effect of an irrelevant object presented only after the saccade on target localization (Deubel et al., 2002, Exp. 2). This experiment used the same stimuli as in Experiment 1however, the pair of distractors was only presented after the saccade. As in the present study, the target reappeared after a blanking period. It turned out that the location of the distractors, present when the eyes land after the primary saccade, was indeed taken by the visual system as the position of the (presaccadic) target. Obviously, the system detecting target position after the end of a saccade is not particularly selective about the geometric characteristics of the stimuli. Visual, form-related features of the reference object are unimportant in searching for the postsaccadic pattern, as long as the location is specified.

Other recent studies have also emphasized the role of postsaccadic references for localization. Honda (1999) showed that visual references can also modify the gain of the presaccadic mislocalization. Lappe, Awater, and Krekelberg (2000) recently studied saccade-induced mislocalization under various conditions. Interestingly, they found that compression of visual space as reported by Ross et al. (1997) occurred only when visual references were available after the saccade. Lappe et al. investigated the time course of the perisaccadic mislocalization of bright stimuli presented briefly before, during, and after the saccade. They compared the localization of these objects in darkness and in the presence of a visible ruler that could serve as a visual reference. Perisaccadic compression of visual space was present only when visual reference was available. Furthermore, in one of their experiments, they presented the ruler either before or after the saccade. Confirming our previous observations (Deubel et al., 2002), their data demonstrate that the ruler acts as a reference even when it is only present 
immediately after the saccade. These findings are strong indications that the "compression of visual space"'-effect reported by Ross et al. (1997) is a consequence of the "reference object" mechanism proposed here and in our previous work, which tries to anchor presaccadically attended objects on the target found after the saccade, rather than the effect of a specific distortion of the spatial representation before and during the saccade.

Does this mean that, under all conditions, the position of any salient object is taken as the target position, discarding relational information from before the saccade? The results from Experiment 2 show that this is clearly not the case. In this experiment, both distractor-target distance and distractor position were varied. The results showed that perceptual localization is now determined mainly by the relational information of target and distractors stored across the saccade, while postsaccadic distractor position per se plays only a minor role. It can be concluded that, if available, relational information in the presaccadic scene is the major determinant of postsaccadic localization. Only if this information is missing, as in the case of the landmark appearing only after the saccade (Deubel et al., 2002) and in the study by Lappe et al. (2000), is the postsaccadic landmark itself taken as the anchor for the localization process.

The findings imply that quite accurate relational information about the relative positions of a few objects in the visual field is stored in a transsaccadic memory and used after a saccade. Evidence in favour of a precise transsaccadic memory of relative spatial positions came from our previous experiments (Deubel et al., 1998), but also from independent work studying the effect of contextual cues on transsaccadic coding of objects. Thus, Verfaillie and de Graef (2000) showed that displacements or rotations of a target that brought it toward another object were easier to detect than changes that moved the target away from another object. Currie et al. (2000) found that detection of a change in location of a saccade target across an eye movement could be made on the basis of both the target's change of absolute position and a change in the spatial relations formed by the target and its neighbours. Carlson-Radvansky (1999) demonstrated that both parts and relational information in scenes composed of geometrical figures are encoded within a fixation, retained in a visual short-term memory across the saccade, and interfere with the postsaccadic processing. Carlson, Covell \& Warapius (2001) used a transsaccadic version of the Jiang, Olson, and Chun (2000) contextual cueing paradigm to show that objects in transsaccadic memory are encoded in relation to one another.

\section{Time constraints for the spatial anchoring}

In the experiments presented here the object that was present when the eyes landed after the saccade was automatically taken as the spatial reference for a target that reappeared only after a blank. This indicates that the 
presence or absence of an object immediately after the saccade is an essential, determining factor for that object to become a spatial reference. Lappe et al. (2000) have also emphasized the importance of the time interval immediately after the saccade for target localization based on visual references. With respect to this issue, Deubel et al. (1998) demonstrated that the question which of two items wins the race to become a reference may be a matter of only a few milliseconds. These experiments used two stimuli, a target and a single distractor. While one of the two was displaced during the saccade, the other was blanked for a short period of time. Even when the postsaccadic gap resulting from the blank was very short (less than 30 $\mathrm{ms}$ ), the blanked object was invariably perceived as moving across the saccade, while the moved (but continuously present) object was perceived as stable. Koch and Deubel (in press) recently reported similar findings from an experiment where both objects appeared with small temporal asynchrony long after the saccade.

\section{Transsaccadic landmarks may also be important for oculomotor accuracy}

The analysis of oculomotor behavior in Experiment 1 demonstrates that secondary, corrective saccades are programmed only on the basis of the reappearing target. This is consistent with an earlier study also showing that when the saccade target is temporarily unavailable after the saccade, corrective saccades tend to wait for target reappearance (Deubel, Wolf, \& Hauske, 1982). However, these findings do not exclude the possibility that relational information stored across the saccade may, under certain conditions, nevertheless be used by the oculomotor system. Hayhoe, Lachter, and Moeller (1992) demonstrate the use of such relational cues in oculomotor spatial accuracy. In a study where the subject's task was to saccade toward two short-duration visual stimuli one of them was lit up again. When the location of the relit stimulus was slightly shifted, the subject's oculomotor localization of the remembered stimulus was also shifted, though, to a smaller extent than the actual shift of the stimulus. The authors conclude that when egocentric and exocentric cues are discordant, oculomotor localization relies on a combination of the two. Karn, Moeller, and Hayhoe (1997) studied the effect of a visual landmark on memory-guided saccades and found that the landmark aided targeting precision. In an investigation by Dassonville, Schlag, and Schlag-Rey (1995), subjects were asked to saccade to perisaccadic stimuli in the presence of a visual stimulus that could provide exocentric location information. Saccadic localization was more accurate in the presence of the landmark, suggesting that localization is based on a combination of exocentric and egocentric cues. 


\section{A modified theory of visual stability around saccadic eye movements}

A number of theories of visual stability have emphasized the special role of the postsaccadic visual lay-out for perceptual stability across saccades. Gibson (1966) and MacKay (1973), for example, had already argued that an actual cancellation of the saccade-induced shifts of the retinal projection by extraretinal signals is unnecessary in normal scenes because visual relationships are preserved during saccades. More recent theories of visual stability have initially focussed on the specific role of the saccade target. Deubel, Wolf, and Hauske (1984) were probably the first to propose that a transsaccadic memory representation of the saccade target may serve to relocate visual objects across saccades. In more recent work, we (Deubel \& Schneider, 1994; Deubel et al., 1996, 1998) developed a reference object theory that assumes that pre- and postsaccadic visual snapshots "snapshots" are linked by means of the saccade target which is assumed by the visual system as being stable. In a very similar theoretical approach, the saccade target theory (Currie et al., 2000; McConkie \& Currie, 1996) also assigned a privileged status to the object that constitutes the target for the saccade.

Both theories assume that with each new fixation the visual system runs through a sequence of processing steps that starts with the selection of one object as the target for the next saccade. Particular features of the saccade target are selected and stored in a transsaccadic memory to facilitate its re-identification at the start of the next fixation. Then the saccade is executed, bringing the target object into central vision. After the eye has landed, the visual system searches for the critical target features within a limited region around the landing site. If the target object is found, the relationship between its retinal location and its mental representation is compared in order to coordinate these two types of information. According to this theory, then, extraretinal signals are not used for transsaccadic integration under normal circumstances, because the reference object usually is found. If the postsaccadic target localization fails (e.g., because the intrasaccadic target shift was too large or the target is absent), however, the assumption of visual stability is abandoned. As a consequence, a target displacement is perceived.

The present findings can now be used to state the "reference object theory" more precisely in several important aspects. The first specification concerns the properties of the spatial window that is considered for localization. The constancy mechanism concentrates on the region near the saccade target, with only secondary influence from other locations - only the saccade goal and possibly a few other attended objects are transferred accurately across saccades (see also, Irwin, McConkie, Carlson-Radvansky, \& Currie, 1994). The results from Experiment 1 (Figure 3) reflect the spatial properties of this "constancy window": it extends, horizontally, to a 
few degrees around the target. Vertically oriented structures are of major importance for the localization process.

A second specification concerns the finding that not just the target, but also other distractor objects can serve as spatial references. Whether an object is defined as target or distractor before the saccade seems to play little role in the postsaccadic determination of the reference object. Critical for the selection of a postsaccadic object as a reference is a temporal constraint, namely its presence right when the eyes land. This demonstrates that temporal continuity of an object is more important even than selection as a saccade target in establishing a reference object.

A third, and very important specification concerns the type of information that is used by the postsaccadic localization process. The experiments demonstrate that the underlying information processing depends on the information that is available for the visual system. Three different scenarios may occur: (1) The "ideal" case occurs when a consistent visual scene is present before and after the saccade-since objects do normally not jump during saccades, this situation conforms the typical case in a natural visual environment. Under this condition the relocalization process is based on the relational information about the different objects in the scene, stored across the saccade (Experiment 2). This information is sufficiently precise and allows anchoring of the transsaccadic memory representation onto the actual, postsaccadic spatial lay-out. So, normally, space constancy depends on comparison of common elements in the pre- and postsaccadic images. (2) If no presaccadic relational information is provided, such as with a target appearing in an otherwise blank field, the visual system tends to accept any localized postsaccadic object (given it appears sufficiently close to the previous target position) as spatial landmark for relocalization (Deubel et al., 2002; Lappe et al., 2000). (3) If no postsaccadic visual information at all is found in the constancy window, as it is the case with target blanking, the assumption of stationarity is broken. Extraretinal signals in combination with a precise memory of the presaccadic target location stored across the saccade are now used to compute the expected target location. Therefore, in the "blanking" condition, intrasaccadic displacements can be detected with considerable accuracy (de Graef \& Verfaillie, 2002; Deubel et al., 1996). Thus, this presaccadic information is stored across the saccade, but normally, when an object is present at the moment the primary saccade lands, it is discarded as soon as the reference object is found.

\section{Possible physiological mechanisms of visual stability across saccadic eye movements}

The mechanisms proposed above are based on the assumption that presaccadic information is remapped over the saccade in order to interact with the post- 
saccadic visual reafference. Neurons in lateral intraparietal cortex (LIP) described by Duhamel, Colby, and Goldberg (1992) may perform some of the computations required by our theory. Receptive fields in this area shift to compensate for a saccade about $80 \mathrm{~ms}$ before the start of the movement. Thus the LIP seems to store presaccadic, visual information across the saccades and possesses quantitative spatial information about the saccade. The receptive fields are large, however, and would not be able to hold details of the features of a reference objects. Similar properties have been reported from neurons in the superior colliculus (Walker, Fitzgibbon, \& Goldberg, 1995).

Further evidence for transsaccadic storage of saccade target features comes from a recent study by Moore, Tolias, and Schiller (1998). These authors studied the visual selectivity of saccade-related responses of area V4 neurons in monkeys making delayed eye movements to receptive field stimuli of varying orientation. The neurons exhibit a selective presaccadic enhancement, quite separate from the response to the stimulus onset. The presaccadic enhancement appears to provide a strengthening of a decaying featural representation immediately before an eye movement is directed to visual targets. The authors suggest that this reactivation provides a mechanism by which a clear perception of the saccade goal can be maintained during the execution of the saccade, possibly for the purpose of establishing perceptual continuity across eye movements. Finally, Olson and colleagues recently provided evidence for the existence of neurons in the supplementary eye field coding specific locations of parts relative to an object across saccadic eye movements (Olson \& Gettner, 1995; Olson, Gettner, Ventura, Carta, \& Kass, 2000).

\section{REFERENCES}

Bischof, N., \& Kramer, E. (1968). Untersuchungen und Überlegungen zur Richtungswahrnehmung bei willkürlichen sakkadischen Augenbewegungen. Psychologische Forschung, 32, 185-218.

Bridgeman, B., Hendry, D., \& Stark, L. (1975). Failure to detect displacement of the visual world during saccadic eye movements. Vision Research, 15, 719-722.

Bridgeman, B., \& Stark, L. (1991). Ocular proprioception and efference copy in registering visual direction. Vision Research, 31(11), 1903-1913.

Bridgeman, B., Van der Heijden, A. H. C., \& Velichkovsky, B. M. (1994). A theory of visual stability across saccadic eye movements. Behavioral and Brain Sciences, 17, 247-292.

Burr, D. C., Morrone, M. C., \& Ross, J. (1994). Selective suppression of the magnocellular visual pathway during saccadic eye movements. Nature, 371, 511-513.

Carlson, L. A., Covell, E. R., \& Warapius, T. (2001). Transsaccadic coding of multiple objects and features. Psychologica Belgica, 41(1/2), 9-27.

Carlson-Radvansky, L. A. (1999). Memory for relational information across saccadic eye movements. Perception and Psychophysics, 61, 919-934.

Crane, H. D., \& Steele, C. M. (1985). Generation V dual-Purkinje-image eye-tracker. Applied Optics, 24, 527-537.

Currie, C. B., McConkie, G. W., Carlson-Radvansky, L. A., \& Irwin, D. E. (2000). The role of the saccade target object in the perception of a visually stable world. Perception and Psychophysics, $62,673-683$.

Dassonville, P., Schlag, J., \& Schlag-Rey, M. (1995). The use of egocentric and exocentric location cues in saccadic programming. Vision Research, 35(15), 2191-2199. 
De Graef, P., \& Verfaillie, P. (2002). Transsaccadic memory for visual object detail. In J. Hyönä, D. Munoz, W. Heide, \& R. Radach (Eds.), The brain's eye: Neurobiological and clinical aspects of oculomotor research (pp. 181-196). Amsterdam: Elsevier Science.

Deubel, H., \& Bridgeman, B. (1995). Fourth Purkinje image signals reveal eye lens deviations and retinal image distortions during saccades. Vision Research, 35(4), 529-538.

Deubel, H., Bridgeman, B., \& Schneider, W. X. (1998). Immediate post-saccadic information mediates space constancy. Vision Research, 38, 3147-3159.

Deubel, H., \& Schneider, W. X. (1994). Can man bridge a gap? Behavioral and Brain Sciences, 17, 259-260.

Deubel, H., \& Schneider, W. X. (1996). Saccade target selection and object recognition: Evidence for a common attentional mechanism. Vision Research, 36, 1827-1837.

Deubel, H., Schneider, W. X., \& Bridgeman, B. (1996). Postsaccadic target blanking prevents saccadic suppression of image displacement. Vision Research, 36(7), 985-996.

Deubel, H., Schneider, W. X., \& Bridgeman, B. (2002). Transsaccadic memory of position and form. In J. Hyönä, D. Munoz, W. Heide, \& R. Radach (Eds.), The brain's eye: Neurobiological and clinical aspects of oculomotor research (pp. 165-180). Amsterdam: Elsevier Science.

Deubel, H., Wolf, W., \& Hauske, G. (1982). Corrective saccades: Effect of shifting the saccade goal. Vision Research, 22, 353-364.

Deubel, H., Wolf, W., \& Hauske, G. (1984). The evaluation of the oculomotor error signal. In A. G. Gale \& F. Johnson (Eds.), Theoretical and applied aspects of eye movement research (pp. 55-62). Amsterdam: Elsevier Science.

Duhamel, J. R., Colby, C., \& Goldberg, M. (1992). The updating of the representation of visual space in parietal cortex by intended eye movements. Science, 225, 90-92.

Foster, D. H., \& Bischof, W. F. (1991). Thresholds from psychometric functions: Superiority of bootstrap to incremental and probit variance estimators. Psychological Bulletin, 109, 152-159.

Germeys, F., de Graef, P., Panis, S., van Eccelpoel, C., \& Verfaillie, K. (2004). Transsaccadic integration of bystander locations. Visual Cognition, 11(2/3), 203-234.

Gibson, J. J. (1966). The senses considered as perceptual systems. Boston: Houghton Mifflin.

Grüsser, O. J., Krizic, A., \& Weiss, L. R. (1987). Afterimage movement during saccades in the dark. Vision Research, 27, 215-226.

Hayhoe, M. M., Lachter, J., \& Moeller, P. (1992). Spatial memory and integration across saccadic eye movements. In K. Rayner (Ed.), Eye movements and visual cognition: Scene perception and reading (pp. 130-145). Berlin: Springer Verlag.

Heywood, S., \& Churcher, J. (1981). Direction-specific and position-specific effects upon detection of displacements during saccadic eye movements. Vision Research, 21, 255-261.

Hoffman, J. E., \& Subramaniam, B. (1995). The role of visual attention in saccadic eye movements. Perception and Psychophysics, 57, 787-795.

Honda, H. (1989). Perceptual localization of visual-stimuli flashed during saccades. Perception and Psychophysics, 45, 162-174.

Honda, H. (1999). Modification of saccade-contingent visual mislocalization by the presence of a visual frame of reference. Vision Research, 39, 51-57.

Irwin, D. E. (1992). Memory for position and identity across eye movements. Journal of Experimental Psychology: Learning, Memory, and Cognition, 18, 307-317.

Irwin, D. E., \& Gordon, R. D. (1998). Eye movements, attention and trans-saccadic memory. Visual Cognition, 5, 127-155.

Irwin, D. E., McConkie, G. W., Carlson-Radvansky, L. A., \& Currie, C. (1994). A localist evaluation solution for visual-stability across saccades. Behavioral and Brain Sciences, 17, 265-266.

Jiang, Y., Olsen, I. R., \& Chun, M. M. (2000). Organization of visual short-term memory. Journal of Experimental Psychology: Learning, Memory, and Cognition, 26, 683-702.

Karn, K. S., Moeller, P., \& Hayhoe, M. (1997). Reference frames in saccadic targeting. Experimental Brain Research, 115, 267-282.

Koch, C., \& Deubel, H. (in press). How visual landmarks affect the detection of stimulus displacement across saccades. Perception. 
Kowler, E., Anderson, E., Dosher, B., \& Blaser, E. (1995). The role of attention in the programming of saccades. Vision Research, 35, 1897-1916.

Lappe, M., Awater, H., \& Krekelberg, B. (2000). Postsaccadic visual references generate presaccadic compression of space. Nature, 403, 892-895.

MacKay, D. M. (1973). Visual stability and voluntary eye movements. In R. Jung (Ed.), Handbook of sensory physiology: Central visual information (pp. 307-331). Berlin: Springer.

Matin, L. (1972). Eye movements and perceived visual direction. In D. Jameson \& L. Hurvitch (Eds.), Handbook of sensory physiology: Visual psychophysics (pp. 331-380). Berlin: Springer.

Matin, E. (1974). Saccadic suppression: A review and an analysis. Psychological Bulletin, 81, 899-917.

Matin, L., Picoult, E., Stevens, J. K., Edwards, M. W., Young, D., \& MacArthur, R. (1982). Oculoparalytic illusion: Visual-field dependent spatial mislocations by humans partially paralyzed with curare. Science, 216, 198-201.

McConkie, G. W., \& Currie, C. B. (1996). Visual-stability across saccades while viewing complex pictures. Journal of Experimental Psychology: Human Perception and Performance, 22, 563-581.

Moore, T., Tolias, A. S., \& Schiller, P. H. (1998). Visual representations during saccadic eye movements. Proceedings of the National Academy of Sciences of the USA, 95(15), 8981-8984.

Olson, C. R., \& Gettner, S. N. (1995). Object-centered direction selectivity in the macaque supplementary eye field. Science, 269, 985-988.

Olson, C. R., Gettner, S. N., Ventura, V., Carta, R., \& Kass, R. E. (2000). Neuronal activity in macaque supplementary eye field during planning of saccades in response to pattern and spatial cues. Journal of Neurophysiology, 84, 1369-1384.

Ross, J., Morrone, M. C., \& Burr, D. C. (1997). Compression of visual space before saccades. Nature, 386, 598-601.

Ross, J., Morrone, C. M., Goldberg, M. E., \& Burr, D. C. (2001). Changes in visual perception at the time of saccades. Trends in Neuroscience, 24, 113-121.

Schlag, J., \& Schlag-Rey, M. (1995). Illusory localization of stimuli flashed in the dark before saccades. Vision Research, 35, 2347-2357.

Schneider, W. X., \& Deubel, H. (2002). Selection-for-perception and selection-for-spatial-motoraction are coupled by visual attention: A review of recent findings and new evidence from stimulus-driven saccade control. In W. Prinz \& B. Hommel (Eds.), Attention and performance XIX: Common mechanisms in perception and action (pp. 609-627). Oxford, UK: Oxford University Press.

Sperry, R. W. (1950). Neural basis of the spontaneous optokinetic response produced by visual inversion. Journal of Comparative Physiological Psychology, 43, 482-489.

Stark, L., \& Bridgeman, B. (1983). Role of corollary discharge in space constancy. Perception and Psychophysics, 34, 371-380.

Verfaillie, K., \& De Graef, P. (2000). Transsaccadic memory for position and orientation of saccade source and target. Journal of Experimental Psychology: Human Perception and Performance, 26, 1243-1259.

Von Helmholtz, H. (1962). Handbuch der Physiologischen Optik. New York: Dover. (Original work published 1866)

Von Holst, E., \& Mittelstaedt, H. (1954). Das Reafferenzprinzip. Wechselwirkungen zwischen Zentralnervensystem und Peripherie. Naturwissenschaften, 20, 464-467.

Walker, M. F., Fitzgibbon, E. J., \& Goldberg, M. E. (1995). Neurons in the monkey superior colliculus predict the visual result of impending saccadic eye-movements. Journal of Neurophysiology, 73, 1988-2003.

Walker, R., Deubel, H., Schneider, W. X., \& Findlay, J. M. (1997). Effect of remote distractors on saccade programming: evidence for an extended fixation zone. Journal of Neurophysiology, 78 , $1108-1119$. 